\title{
A Monte Carlo Study of Distribution Function $P(S)$ of a Polymer Chain ${ }^{\dagger}$
}

\author{
Mengbo Luo, ${ }^{*}$ Xubing SonG, ${ }^{* *}$ Wenqin LU, ${ }^{*}$ and Jianmin $\mathrm{XU}^{*, \dagger \dagger}$ \\ * Department of Physics, Hangzhou University, Hangzhou 310028, \\ People's Republic of China \\ ** Department of Chemistry, Hangzhou University, Hangzhou 310028, \\ People's Republic of China
}

(Received January 11, 1995)

\begin{abstract}
The distribution function $P(S)$ of the radius of gyration of linear polyethylene (PE) is studied by use of Monte Carlo method in tetrahedral lattice. Three different cases, according to different interactions between polymer chain molecules, are investigated. The $P(S)$ s of three different cases are compared with the exact distribution function $P_{0}(S)$ for random flight chain obtained by Yamakawa. At $\theta$-state, the $P(S)$ of $N=260$ ( $N$ is the length of polymer chain) is similar to $P_{0}(S)$, but that of $N=20$ deviates largely from $P_{0}(S)$. When the chain perturbed by an excluded volume, the difference between $P(S)$ and $P_{0}(S)$ becomes large, but when we introduce long-range interaction (Van der Waals potential) at the same time, the difference becomes smaller. The results show that not only can short chain effect affect the distribution function $P(S)$, excluded volume and long-range interaction can also affect $P(S)$ of the chain.

KEY WORDS Monte Carlo Method / Distribution Function / Radius of Gyration / Short-Range Interaction / Long-Range Interaction /
\end{abstract}

The theoretical study of the conformational characteristics of polymer chain is one of the most important subjects in polymer science. Saying conformation we often mean the spatial shapes of polymer chains, which are customarily described by the mean-square end-toend distance $\left\langle R^{2}\right\rangle$, the mean-square radius of gyration $\left\langle S^{2}\right\rangle$, and the distribution function $P(R)$ and $P(S)$.

The $P(R)$ s of polymer chains have long been studied, ${ }^{1-4}$ the $P(R)$ of polymethylene chain with a short number of bonds $(N=10)$ can even be given accurately. ${ }^{5}$ Resently, Bishop et al. ${ }^{6}$ have investigated the influence of excluded volume on $P(R)$, Rubio et $a l^{7}$ have studied the $P(R)$ between any pair of units in polymer chains by an off-lattice Monte Carlo method.

The investigations in $P(S)$ have being carried on since it was first studied by Fixman, ${ }^{8-10}$ and Flory-Fisk ${ }^{11}$ expressed the $P(S)$ of a polymer chain unperturbed by excluded volume by use of the empirical expression:

$$
P(S)=\text { const } \cdot S^{6} \cdot \exp \left(-7 S^{2} / 2\left\langle S^{2}\right\rangle_{0}\right)
$$

The exact expression of $P(S)$ obtained by Yamakawa $^{12}$ (hereafter referred to simply as $\left.P_{0}(S)\right)$ is:

$$
\begin{aligned}
& P_{0}(S)= \\
& \quad \frac{1}{2^{1 / 2} \pi\left\langle S^{2}\right\rangle^{1 / 2} t^{3}} \sum_{k=0}^{\infty} \frac{(2 k+1) !}{\left(2^{k} k !\right)^{2}}(4 k+3)^{7 / 2} \\
& \quad \times \exp \left(-t_{k}\right) \times\left[\left(1-\frac{5}{8 t_{k}}\right) K_{1 / 4}\left(t_{k}\right)\right. \\
& \left.\quad+\left(1-\frac{3}{8 t_{k}}\right) K_{3 / 4}\left(t_{k}\right)\right]
\end{aligned}
$$

where $K_{s}$ are the modified Bessel functions of the second kind and $t_{k}$ is defined by

\footnotetext{
$\dagger$ This work was supported by the National Science Foundation of Zhejiang Province, China.

$\dagger^{\dagger}$ To whom all correspondence should be addressed.
} 


$$
t_{k}=\frac{(4 k+3)^{2}}{8 t}
$$

while

$$
t=S^{2} /\left\langle S^{2}\right\rangle
$$

For small $t$, by use of asymptotic expansion of $K_{1 / 4}$ and $K_{3 / 4}$, one obtain

$$
\begin{aligned}
P_{0}(S)= & 18\left(\frac{6}{\pi\left\langle S^{2}\right\rangle}\right)^{1 / 2} t^{-5 / 2} \exp \left(-\frac{9}{4 t}\right) \\
& \times\left(1-\frac{19}{36} t+\frac{105}{1296} t^{2}-\cdots\right)
\end{aligned}
$$

Fujita and Norisuye ${ }^{13}$ obtained the asymptotic expansion of $P_{0}(S)$ valid for large $t$, the result is

$$
\begin{aligned}
P_{0}(S) & =\frac{2^{1 / 2} \pi^{5 / 2} t}{\left\langle S^{2}\right\rangle^{1 / 2}} \exp \left(-\frac{\pi^{2} t}{4}\right) \\
\times & {\left[1+\frac{9}{4 \pi^{2}}\left(\frac{1}{t}\right)+\frac{8 \pi^{2}+15}{32 \pi^{4}}\left(\frac{1}{t}\right)^{2}+\cdots\right] }
\end{aligned}
$$

Resently, Linxi and Jianmin ${ }^{14}$ studied the $P(S)$ of unperturbed linear polymer chains by using Monte Carlo method in the simple cubic lattice. They found a slight difference between their $P(S)$ and $P(S)$ of Flory (eq 1), but very similar to $P_{0}(S)$.

But all these previous studies on $P(S)$ dealt with some simplified model chain, such as random flight chain, self-avoiding chain on simple cubic lattice, etc., they didn't consider the spatial configuration of a real polymer chain. In the present work the $P(S)$ of a real polymer chain, linear polyethylene (PE) chain, in dilute solution is studied. We simulate the configurations of PE by use of Monte Carlo method in tetrahedral lattice, restricted to a three rotational isomeric states (RIS) model, and submitted to short-range interactions and long-rang interactions. ${ }^{15,16}$

\section{METHOD OF CALCULATION}

The length of the $\mathrm{C}-\mathrm{C}$ bond and the valence angle of the CCC are fixed as $1.53 \AA$ and $109.5^{\circ}$, respectively. The bond can only occur in three ways, trans $\left(\phi=0^{\circ}\right)$, gauche ${ }^{+}\left(\phi=120^{\circ}\right)$ and gauche $^{-}\left(\phi=240^{\circ}\right)$. The primary statistical weight matrix is ${ }^{1}$

$$
U=\stackrel{t}{g^{+}} g^{-}\left[\begin{array}{ccc}
t & g^{+} & g^{-} \\
1 & \sigma & \sigma \\
1 & \sigma & \sigma \omega \\
1 & \sigma \omega & \sigma
\end{array}\right]
$$

here $\sigma=\exp \left(-E_{\sigma} / k T\right), \omega=\exp \left(-E_{\omega} / k T\right)$, and $k$ is the Boltzmann constant.

Refering to Abe et al. ${ }^{17}$ we choose $E_{\sigma}=500$ cal mol ${ }^{-1}$ and $E_{\omega}=2500 \mathrm{cal} \mathrm{mol}^{-1}$ the temperature $T=410 \mathrm{~K}$.

The long-range interactions between nonneighbor molecules $k$ and $l$ can be calculated by the formula ${ }^{17}$

$$
E_{k l}=a_{k l} \cdot \exp \left(-b_{k l} r_{k l}\right)-c_{k l} / r_{k l}^{6}
$$

$r_{k l}$ is the distance between two molecules.

Simply, we only consider the carbon-carbon interaction, the value of $a_{k l}$ is $9.086 \times 10^{5}, b_{k l}$ is $4.59, c_{k l}$ is 363.0 , and $E_{k l}$ is in $\mathrm{kcal} \mathrm{mol}^{-1}$ when $r_{k l}$ in $\AA$.

Three different situations of the chain are considered in this paper. They correspond respectively to three different interactions between polymer chain molecules, three different potentials are used to represent these interactions in this paper. ${ }^{18}$

Case A: Only short-range interactions are considered, the chain is at $\theta$ state.

Case B: An excluded volume is adopted to simulate the long-range interactions of chain, this situation corresponds to the chains solved in a good solvent, the chain is in an expending conformation. In this paper, the diameter of excluded volume is chosen arbitrarily to be $2.5 \AA$, the value is changeable for different solvents and different temperatures.

Case C: A Van der Waals potential and a excluded volume are together adopted to simulate the long-rang interactions, when the chains solved in a poor solvent. Formular 8 is adopted to represent the Van der Waals 
potential in our calculation. After adopted a Van der Waals potential, the chain could be in a more folding conformation compared to case B.

In a growing polymer chain, the next bond can occur in three ways: $t, g^{+}$, and $g^{-}$. The energies $\left(E_{t}, E_{g^{+}}, E_{g^{-}}\right)$of the next three states are calculated in case A, B, and C. The probabilities of these states are:

$$
\begin{aligned}
& P_{t}= \\
& \frac{\exp \left(\frac{-E_{t}}{k T}\right)}{\exp \left(\frac{-E_{t}}{k T}\right)+\exp \left(\frac{-E_{g^{+}}}{k T}\right)+\exp \left(\frac{-E_{g^{-}}}{k T}\right)}
\end{aligned}
$$

$P_{g^{+}}=$

$$
\frac{\exp \left(\frac{-E_{g^{+}}}{k T}\right)}{\exp \left(\frac{-E_{t}}{k T}\right)+\exp \left(\frac{-E_{g^{+}}}{k T}\right)+\exp \left(\frac{-E_{g^{-}}}{k T}\right)}
$$

$$
\begin{aligned}
& P_{g^{-}}= \\
& \frac{\exp \left(\frac{-E_{g^{-}}}{k T}\right)}{\exp \left(\frac{-E_{t}}{k T}\right)+\exp \left(\frac{-E_{g^{+}}}{k T}\right)+\exp \left(\frac{-E_{g^{-}}}{k T}\right)}
\end{aligned}
$$

Then the next state is chosen at random, but when its coordinate is within the excluded volume, it is eliminated.

If $W(S)$ is the Rosenbluth-Rosenbluth probability $^{19}$ ( $\mathrm{R}-\mathrm{R}$ probability) of a sample which radius gyration is $S$, we have

$$
\int_{S}^{S+\Delta S} P(S) \delta S=\frac{\sum_{S=S}^{S+\Delta S} W(S)}{\sum_{S=0}^{\infty} W(S)}
$$

where $\sum_{S+S}^{S+\Delta S} W(S)$ is the sum of the $\mathrm{R}-\mathrm{R}$ probabilities of all the samples whose radius gyrations lie between $S$ and $S+\Delta S$.
If $\Delta S$ is small enough, the left part of eq 12 can be written as $P(S) \cdot \Delta S$, then

$$
P(S)=\frac{\sum_{S=S}^{S+\Delta S} W(S)}{\Delta S \cdot \sum_{S=0}^{\infty} W(S)}
$$

In our calculation, $\Delta S$ is equal to $0.025\left\langle S^{2}\right\rangle^{1 / 2}$.

\section{RESULTS OF MONTE CARLO COMPUTATIONS}

Monte Carlo simulations are carried out for chains of length $N$ from 20 to 260 bonds at the temperature $410 \mathrm{~K}$, and we use 15000 samples.

In Figures 1 and $2, \log \left\langle R_{N}^{2}\right\rangle$ and $\log \left\langle S_{N}^{2}\right\rangle$ is plotted versus $\log N$ for three different cases,

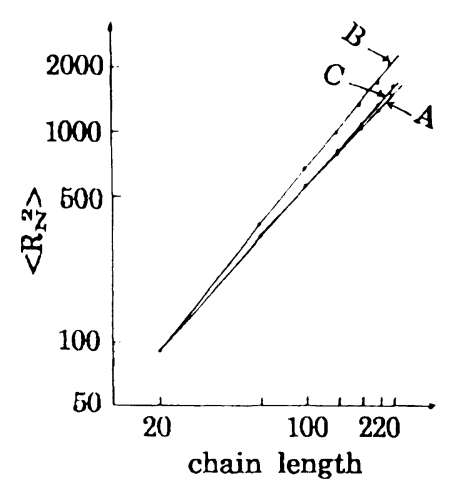

Figure 1. Log-log plot of mean square end-to-end distance $v s$. chain length $N$ of case A, case B, and case C. $N=20,60,100,140,180,220$, and 260 .

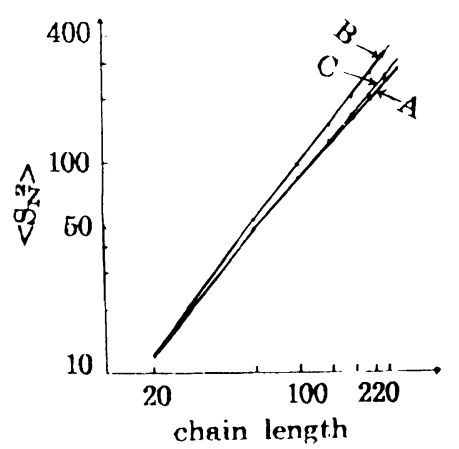

Figure 2. Log-log plot of mean-radius gyration vs. chain length of case $\mathrm{A}, \mathrm{B}$, and $\mathrm{C}$. 
M. Luo et al.

Table I. Dimensions and scaling law exponent data for different chain lengthes and situations ${ }^{\mathbf{a}}$

\begin{tabular}{|c|c|c|c|c|c|c|}
\hline \multirow{2}{*}{$N$} & \multicolumn{3}{|c|}{$\left\langle R^{2}\right\rangle / l^{2}$} & \multicolumn{3}{|c|}{$\left\langle S^{2}\right\rangle / l^{2}$} \\
\hline & A & B & $\mathrm{C}$ & A & B & $\mathrm{C}$ \\
\hline 20 & 89.99 & 92.26 & 88.12 & 11.78 & 11.96 & 11.63 \\
\hline 60 & 320.57 & 359.23 & 315.04 & 47.82 & 52.14 & 46.96 \\
\hline 100 & 548.05 & 659.11 & 549.72 & 85.42 & 99.39 & 84.70 \\
\hline 140 & 773.32 & 967.99 & 774.90 & 123.26 & 149.49 & 123.25 \\
\hline 180 & 997.54 & 1290.65 & 1011.74 & 160.94 & 202.32 & 162.04 \\
\hline 220 & 1215.62 & 1640.58 & 1268.17 & 198.48 & 257.86 & 202.19 \\
\hline 260 & 1443.61 & 1994.73 & 1522.02 & 236.02 & 312.54 & 243.46 \\
\hline$\gamma_{R} \gamma_{S}$ & 1.03 & 1.17 & 1.08 & 1.06 & 1.22 & 1.12 \\
\hline$A_{R} A_{S}$ & 4.77 & 3.01 & 3.80 & 0.639 & 0.487 & 0.361 \\
\hline
\end{tabular}

${ }^{a} l$ is the length of carbon-carbon bond.

respectively. These plots all become linear for large $N(N \geq 60)$, implying that long flexible chains always obey the scaling law:

$$
\begin{aligned}
& \left\langle R_{N}^{2}\right\rangle=A_{R} \cdot N^{\gamma_{R}} \\
& \left\langle S_{N}^{2}\right\rangle=A_{S} \cdot N^{\gamma_{S}}
\end{aligned}
$$

no matter in which situation the chain be. It will be observed that the plots of case $C$ are closer to those of case A than case B to case $\mathrm{A}$, this means that long-range attraction can compensate the excluded volume. If the attraction is strong enough, one could find that the chain of case $\mathrm{C}$ will adopt more folding conformations.

The $\left\langle R_{N}^{2}\right\rangle$ and $\left\langle S_{N}^{2}\right\rangle$ of different lengthes and the scaling low exponent $\gamma_{R}$ and $\gamma_{S}$ are collected in Table I. The $\gamma_{R}$ and $\gamma_{S}$ of case A approximate to 1.0 that indicate the simulation of $\theta$-state is quite correct.

In Figure 3, we show distribution of $N=20$ and $N=260$ of case $\mathrm{A}$, the $P_{0}(S)$ (eq 2) is also showed. Since the distributions $N=60,100$, up to 220 are within that of $N=20$ and $N=260$, so these distributions are not shown. The deviation is very significant for short length chain, however it becomes small for long chain. This is because the polyethylene chain isn't a random flight chain, which submitted to shortrange interaction, there exists short chain

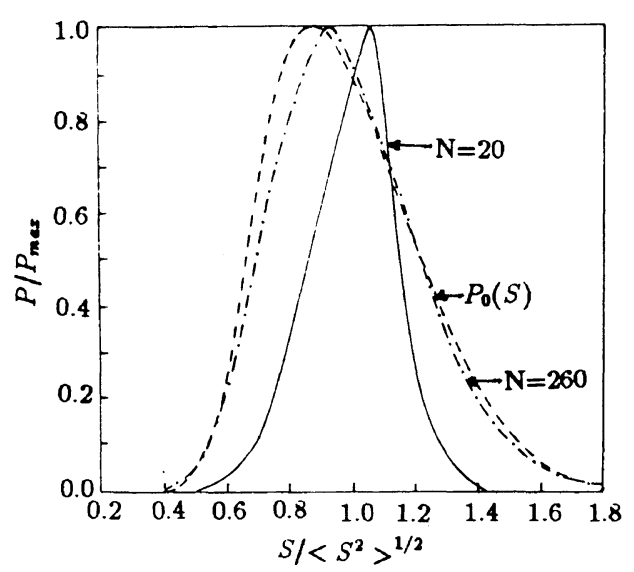

Figure 3. A comparison of the distribution (relative to their maximum value) for the radius of gyration $S$ of the case $\mathrm{A}$ and of the random flight chain. Dashed line, $P_{0}(S)$; solid line, of $N=20$; dot dashed line, of $N=260$.

effect on the chain. This effect affects greatly the short length chain, but is slowly eliminated when the chain length increases. ${ }^{1}$

In Figure 4, we show the $P(S)$ results obtained for case B of chain length 20 and 260. The difference between $P(S)$ and $P_{0}(S)$ of case $\mathrm{B}$ are bigger than that of case $\mathrm{A}$, this indicates that the excluded volume also affects the distribution $P(S)$ as well as it affects $\left\langle S^{2}\right\rangle, \gamma_{R}$, $\gamma_{S}$, etc. The $P(S)$ s of case B may change for different diameter of excluded volume, if the diameter equals 0 , the $P(S)$ s of case B become 


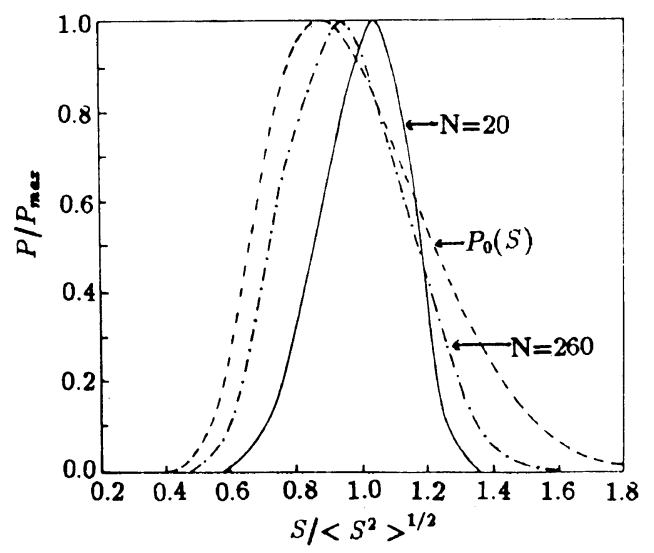

Figure 4. A comparison of the distribution (realtive to their maximum value) for the radius of gyration $S$ of the case $\mathrm{B}$ and of the random flight chain. Dashed line, $P_{0}(S)$; solid line, of $N=20$; dot dashed line, of $N=260$.

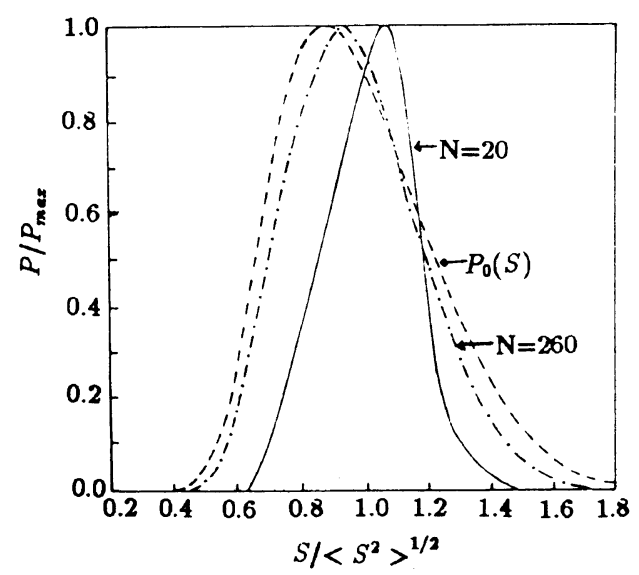

Figure 5. A comparison of the distribution (relative to thir maximum value) for the radius of gyration $S$ of the case $\mathrm{C}$ and of the random fiight chain. Dashed line, $P_{0}(S)$; solid line, of $N=20$; dot dashed line, of $N=260$.

that of case A.

In Figure 5, the $P(S)$ s of chain length 20 and 260 are shown. When we introduce long-range interaction, whose primary part is long-range attractive since there exists an excluded volume at the same time, the difference between $P(S)$ and $P_{0}(S)$ becomes smaller than that of case B. One could also find the curves of $N=260$ are very alike in shape for both situations of case $\mathrm{A}$ and $\mathrm{C}$. Like case $\mathrm{B}$, the $P(S)$ s of case $\mathrm{C}$ will also change for different diameter of excluded volume and different interaction potential. Obviously, one can simulate $\theta$-state of polymer chain by changing the diameter of excluded volume or long-range interaction potential.

One could find that the peaks of $P(S)$ of three cases occur at nearly the same value of $S /\left\langle S^{2}\right\rangle^{1 / 2}$ at the same chain length $N$ though $\left\langle S^{2}\right\rangle$ s are different for these cases, and the peak of $P(S)$ moves toward that of $P_{0}(S)$ when the length increases. It suggests that the location of the peak of $P(S)$ is determined mainly by the chain length, and indicates the peak of $P(S)$ of an infinity chain will coincide with that of $P_{0}(S)$.

In addition, we also calculated the reduced moment

$$
\delta(p, 2)=\left\langle S^{p}\right\rangle /\left\langle S^{2}\right\rangle^{p / 2}
$$

for $p=4,6,8,10$, and 12 . The results are listed in Table II, $\delta(p, 2)$ s of random flight chain obtained by Yamakawa ${ }^{12}$ (hereafter referred to simply as $\delta_{0}(p, 2)$ s) are also shown. $\delta(p, 2) \mathrm{s}$ of case A increase as chain length increases, and all asymptotical to $\delta_{0}(p, 2) \mathrm{s}$. But the $\delta(p, 2)$ s of case $\mathrm{B}$ and $\mathrm{C}$ show large deviation from $\delta_{0}(p, 2)$ s, and they don't always increase as chain length increases, relatively, the derivation of case $\mathrm{C}$ is slightly smaller.

Even in the case $\mathrm{A}$, the $\delta(p, 2)$ s show greatly dependence on chain length $N$, opposite to the results obtained by $\operatorname{LinXi^{14}}$ that $\delta(p, 2)$ s are approximately equal to $\delta_{0}(p, 2)$ s for all length. This difference must come from using different models, we consider the configuration energy $\left(E_{t}, E_{g^{+}}, E_{g^{-}}\right)$in the tetrahedral lattice, while the later didn't consider the energy in the simple cubic lattice. After considering the configuration energy, the chain manifests stiffer than not, then the deviation from random flight chain becomes more significant.

The chain in the case B is in an expanding conformation relatively to the chain in the case A, but the $\delta(p, 2) \mathrm{s}$ in the case $\mathbf{B}$ are always smaller than those in the case A. Generally, affected by an excluded volume, a configuation 
M. Luo et al.

Table II. Numerical results of $\delta(p, 2)$ for different chain lengthes and situations

\begin{tabular}{|c|c|c|c|c|c|c|c|c|c|}
\hline$p$ & $N=$ & 20 & 60 & 100 & 140 & 180 & 220 & 260 & $\delta_{0}(p, 2)^{\mathrm{a}}$ \\
\hline \multirow{3}{*}{4} & A & 1.065 & 1.160 & 1.200 & 1.217 & 1.225 & 1.230 & 1.235 & \multirow{3}{*}{1.267} \\
\hline & B & 1.056 & 1.124 & 1.151 & 1.168 & 1.174 & 1.172 & 1.166 & \\
\hline & $\mathrm{C}$ & 1.063 & 1.153 & 1.187 & 1.200 & 1.207 & 1.209 & 1.210 & \\
\hline \multirow{3}{*}{6} & A & 1.193 & 1.518 & 1.679 & 1.755 & 1.791 & 1.815 & 1.844 & \multirow{3}{*}{2.003} \\
\hline & B & 1.168 & 1.395 & 1.500 & 1.578 & 1.601 & 1.591 & 1.569 & \\
\hline & $\mathrm{C}$ & 1.190 & 1.500 & 1.646 & 1.700 & 1.739 & 1.747 & 1.751 & \\
\hline \multirow{3}{*}{8} & A & 1.394 & 2.189 & 2.672 & 2.915 & 3.037 & 3.126 & 3.246 & \multirow{3}{*}{3.870} \\
\hline & B & 1.346 & 1.881 & 2.179 & 2.423 & 2.504 & 2.465 & 2.400 & \\
\hline & $\mathrm{C}$ & 1.393 & 2.157 & 2.610 & 2.776 & 2.933 & 2.978 & 2.974 & \\
\hline \multirow{3}{*}{10} & A & 1.690 & 3.413 & 4.726 & 5.431 & 5.815 & 6.106 & 6.549 & \multirow{3}{*}{8.920} \\
\hline & B & 1.606 & 2.716 & 3.461 & 4.160 & 4.418 & 4.287 & 4.108 & \\
\hline & $\mathrm{C}$ & 1.696 & 3.363 & 4.635 & 5.115 & 5.670 & 5.915 & 5.830 & \\
\hline \multirow{3}{*}{12} & A & 2.116 & 5.674 & 9.135 & 11.12 & 12.30 & 13.22 & 14.76 & \multirow{3}{*}{23.193} \\
\hline & B & 1.978 & 4.346 & 5.925 & 7.852 & 8.663 & 8.230 & 7.757 & \\
\hline & $\mathrm{C}$ & 2.136 & 5.599 & 9.054 & 10.45 & 12.32 & 13.58 & 12.98 & \\
\hline
\end{tabular}

${ }^{a}$ Calculated from eq 8.43 of Yamakawa. ${ }^{12}$

be in the case A previously will expand, then the square radius of gyration $S^{2}$ will become larger. But this effect affects differentially on different $S^{2}$. For a small $S^{2}$ in the case A, some segments of the chain are very close to each other and some even intervene; while for a large $S^{2}$, such segments are relatively fewer. So, when the chain perturbed by an excluded volume, the incrementary ratio $\Delta S^{2} / S^{2}$ of a previous small $S^{2}$ will be larger than that of a previous large $S^{2}$. For this reason, after introducing an excluded volume, the incrementary ratio for $\left\langle S^{2}\right\rangle$ will be larger than for $\left\langle S^{p}\right\rangle$ $(p>4), \delta(p, 2)$ decreases while $\left\langle S^{2}\right\rangle$ increases.

The absolute width of distribution $S_{\max }-$ $S_{\min }$ is calculated for these three cases, they are listed in Table III. The values of case A are bigger than that of case $\mathrm{C}$ for the same chain length, while the values of case $\mathrm{C}$ bigger than that of case $\mathrm{B}$, but $\left\langle S^{2}\right\rangle$ of these cases are just opposite.

When we deal with a real polymer chain (PE chain) at $\theta$-state (case $\mathrm{A}$ ), which only submitted to short-range interaction, there exist some differences between $P(S)$ and $P_{0}(S)$ of random
Table III. The absolute width of distribution of different chain lengthes for three cases

\begin{tabular}{rrrr}
\hline & \multicolumn{3}{c}{$\left(S_{\max }-S_{\min }\right) / l$} \\
& \multicolumn{1}{c}{$\mathrm{A}$} & \multicolumn{1}{c}{$\mathrm{B}$} & $\mathrm{C}$ \\
\hline 20 & 3.10 & 2.77 & 2.90 \\
60 & 8.30 & 7.22 & 7.70 \\
100 & 12.48 & 11.22 & 11.50 \\
140 & 15.27 & 14.06 & 14.15 \\
180 & 18.08 & 15.65 & 17.19 \\
220 & 20.43 & 17.66 & 19.20 \\
260 & 23.04 & 19.45 & 21.06
\end{tabular}

${ }^{\mathrm{a}} l$ is the length of carbon-carbon bond.

flight chain, but the differences will become smaller and smaller as chain length increases, for the differences caused by short chain effects. When we introduce an excluded volume (case B), the differences are large even for long chain. But when we introduce Van der Waals potential at the same time, the deviation from $P_{0}(S)$ at case $\mathrm{C}$ becomes much smaller than case $B$. The excluded volume and long-range attraction not only change $\left\langle S^{2}\right\rangle, \gamma_{R}, \gamma_{S}$ of chain, they also affect the distribution $P(S)$ of 
the radius of gyration. $P(S)$ will be varied with different excluded volume and attraction, this will be studied next. Besides, the chain of case $\mathrm{C}$ will be in a more folding conformation even than that of case $\mathrm{A},{ }^{20,21} P(S)$ of that situation need to be studied.

\section{REFERENCES}

1. P. J. Flory, "Statistical Mechanics of Chain Molecules," Wiley, New York, N.Y., 1969.

2. S. Redner, J. Phys., A, 13, 3525 (1980).

3. M. Fixman and R. Alben, J. Chem. Phys., 58, 1553 (1973).

4. D. Y. Yoon and P. J. Flory, J. Chem. Phys., 61, 5366 (1974).

5. J. Freire and M. Fixman, J. Chem. Phys., 69, 634 (1978).

6. M. Bishop, J. H. R. Clarke, A. Rey, and J. J. Freire, J. Chem. Phys., 95, 4589 (1991).

7. A. M. Rubio, J. J. Freire, M. Bishop, and J. H. R. Clarke, Macromolecules, 26, 4018 (1993).
8. M. Fixman, J. Chem. Phys., 36, 306 (1962).

9. C. Domb, J. Gillis, and G. Wilmers, Proc. Phys. Soc., 85, 625 (1965).

10. R. F. Hoffman and W. C. Forsman, J. Chem. Phys., 50, 2316 (1969).

11. P. J. Flory and S. Fisk, J. Chem. Phys., 44, 2243 (1966).

12. H. Yamakawa, "Modern Theory of Polymer Solutions," Interscience, New York, N.Y., 1971.

13. H. Fujita and T. Norisuye, J. Chem. Phys., 52, 1115 (1970).

14. Z. LinXi and X. JianMin, Polym. J., 22, 426 (1990).

15. M. A. Winnik, D. Rigby, R. F. T. Stepto, and B. Lemaire, Macromolecules, 13, 699 (1980).

16. J. Xu, X. Son, and Z. Zhou, Eur. Polym. J., 25, 601 (1989).

17. A. Abe, R. L. Jernigan, and P. J. Flory, J. Am. Chem. Soc., 88, 631 (1966).

18. O. Collet and S. Premilat, Macromolecules, 26, 6076 (1993).

19. M. N. Rosenbluth and A. W. Rosenbluth, J. Chem. Phys., 23, 356 (1955).

20. I. Webman, J. L. L. Lebowitz, and M. H. Kalos, Macromolecules, 14, 1495 (1981).

21. I. C. Sanchez, Macromolecules, 15, 549 (1982). 